\title{
Recruitment of Black Men and Women into Academic Psychiatry
}

\author{
Lia A. Thomas ${ }^{1,2}$ (1) - Richard Balon ${ }^{3} \cdot$ Eugene V. Beresin $^{4} \cdot$ John Coverdale ${ }^{5} \cdot$ Adam M. Brenner $^{1}$ • \\ Anthony P. S. Guerrero ${ }^{6}$ - Alan K. Louie ${ }^{7}$. Laura Weiss Roberts ${ }^{7}$
}

Received: 10 June 2019 / Revised: 10 June 2019 / Accepted: 19 June 2019 / Published online: 24 July 2019

(C) This is a U.S. government work and its text is not subject to copyright protection in the United States; however, its text may be subject to foreign copyright protection 2019

Academic psychiatry is a discipline of medicine and a mental health profession dedicated to helping others live their best lives despite the burdens and anguish associated with having a mental disorder. In clinical work, academic psychiatrists embrace all people from all walks of life. In advocacy work, academic psychiatrists fight for the rights and well-being of people who often experience marginalization, underrepresentation, and stigma. And yet, the workforce of the field of academic psychiatry is not representative of the people and populations it serves. The demographic makeup of academic psychiatry, while increasingly balanced with respect to gender, remains disproportionately white [1].

Accreditation bodies of medical schools have instituted policies speaking to the need for diversity in the student body, staff, and faculty and for education in cultural competence [2]. Most recently, the Accreditation Council for Graduate Medical Education added the following to its core program requirements [3]:

The program, in partnership with its Sponsoring Institution, must engage in practices that focus on mission-driven, ongoing, systematic recruitment and retention of a diverse and inclusive workforce of residents,

Lia A. Thomas

Lia.Thomas@utsouthwestern.edu

1 University of Texas Southwestern Medical Center, Dallas, TX, USA

2 VA North Texas Health Care System, Dallas, TX, USA

3 Wayne State University, Detroit, MI, USA

4 Harvard Medical School, Boston, MA, USA

5 Baylor College of Medicine, Houston, TX, USA

6 University of Hawai'i John A. Burns School of Medicine, Honolulu, HI, USA

7 Stanford University, Stanford, CA, USA fellows (if present), faculty members, senior administrative staff members, and other relevant members of its academic community. (p. 5)

Numerous groups of persons are considered underrepresented in medicine. The Association of American Medical Colleges (AAMC) [4] defines underrepresented in medicine as "racial and ethnic populations that are underrepresented in the medical profession relative to their numbers in the general population." The AAMC identifies Blacks, MexicanAmericans, Native Americans (including American Indians, Alaska Natives, and Native Hawaiians), and mainland Puerto Ricans as underrepresented [4].

Black men and women are poorly represented in medicine. In the most recent AAMC survey of US faculty, approximately 6200 US medical school faculty members identified as Black [1]. With over 170,000 faculty members identified, the percentage equates to 3.5 . In the last US census, $13 \%$ of the US population identified as African American [5].

In academic psychiatry, the numbers are similarly disheartening. That same AAMC survey [1] noted that in psychiatry, 141 Black men and 270 Black women were on faculty nationwide. Lett et al. [6] noted that in comparison to the US census numbers, Black and Hispanic physicians in academic departments were less well represented in 2016 than in 1990. Two influential reports $[7,8]$ have spoken on the need to diversify the workforce in order to address health care disparities and on the need for more minority faculty. These reports discuss the importance of a diverse and inclusive workforce as a means to reduce health care disparities and to decrease the implicit and explicit biases that exist in the US health care system. For specific populations, having clinicians of one's racial or ethnic group may more effectively destigmatize psychiatric disorders than destigmatizing efforts from outside one's racial or ethnic community.

We must think strategically about ways to rectify these deficits and to improve the recruitment and retention of individuals of African descent or who identify as African 
Americans into psychiatry. There needs to be an assessment of diversity needs and a fostering of interest among women and men of African descent or who identify as African American in psychiatry. We will discuss some relevant strategies and highlight the role of academic psychiatrists in these processes.

Recognizing that the terms of African descent or African American do not fully encompass the cultural community within the USA, we instead use the term Black throughout this editorial. This phrasing is in keeping with the 2019 book Black Mental Health: Patients, Providers, and Systems, edited by Ezra Griffith, Billy Jones, and Altha Stewart [9]. The term encompasses physicians with family roots that trace back to before the Civil War, physicians who are first generation immigrants from African countries, and physicians who identify their Latino roots with equal importance.

\section{Assessment of Needs}

The diversity needs in existing communities should be identified systematically and specifically. We suggest that academic centers should take a serious inventory of the current and future demographics within their institutions and the communities in which they practice. The needs of Detroit, Michigan, for example, will differ from those of El Paso, Texas, which, in turn, will differ from those of Portland, Oregon. In addition, even within different urban and rural centers, demographic and cultural differences may occur within certain parts of cities, towns, and rural neighborhoods.

To perform such attuned and rigorous assessments, it will be essential to ask and seek answers to challenging questions about why the demographics of the faculty do not match the demographics of the communities in which they practice. In one survey [10], women and minority faculty left a medical school for reasons related to other opportunities for career and professional advancement, low salaries, and chair and departmental factors. To dive deeply into the issues surrounding recruitment and retention, we encourage surveying current faculty and arranging for exit interviews of departing faculty.

We suggest that it is also critical to examine structural barriers that limit opportunities for Black women and men to be recruited and retained. We should endeavor to identify biases, including implicit biases in hiring physicians of color, LGBTQ physicians, and other physicians who are not among the majority. Those who recruit and hire especially should aim to become aware of any such biases and perhaps receive training in the use of the Implicit Association Test and other means [11] to minimize the influence of these biases.
Fostering an Interest in Psychiatry at Multiple Levels

Recruitment of Black men and women into psychiatry requires those currently in the field to invest effort at multiple points along the workforce pipeline and, in light of the observation that numbers of Black women matriculants in medical school are increasing (though still insufficient), to explore how gender may shape experiences along this pipeline. Middle and high school students, who may be vulnerable to discrimination, limited exposure to career options, and other socioeconomic adversities that may derail future education, need to be encouraged to pursue careers in health care. Examples of these programs include the Health Professions Recruitment and Exposure Program (HPREP) at institutions like University of Texas Southwestern, Yale, and University of Chicago [12-14]. These pipeline programs introduce students to careers in medicine and provide the opportunity to network with medical students and attending physicians. College students will also benefit from mentoring and exposure to health care work opportunities. We would encourage academic psychiatrists to look not only at their undergraduate colleges affiliated with their institutions but also at other colleges surrounding their institutions. Bright, interested students of color may be close by and in need of mentoring and support. We encourage academic psychiatrists to look beyond the expected internal candidates.

A pressure to conform in medical school or to become part of the "culture of medicine" can diminish or quash a celebration of individual differences [15]. Students should not succumb to pressures to become a stereotypical doctor or to spend their lifetime becoming something they are not [16]. Professionalism is best served by a health care culture that is diverse, attentive, and responsive to the needs of patients from varying backgrounds. The hidden curriculum, with messages such as "don't stand out," "don't make too much noise," and "don't be different from the other students," can subvert individual differences and diminish the good that individuals can do in our field.

Humanities courses in medical school can serve as two-way conversations about race and culture. Examples of curricula on cultural humility, which promote discussions about race and which focus on addressing injustices and disparities in health care, should be shared as models for undergraduate and graduate medical education and faculty development [17-19]. Regional partnerships, such as one described by Harris et al. [20], can benefit trainees, faculty, and institutions alike. Partnerships should be fostered between academic psychiatry organizations, including the American Association of Directors of Psychiatric Residency Training and the Association of Directors of Medical Student Education in Psychiatry, and underrepresented minority medical student organizations, including 
the Student National Medical Association and the Latino Medical Student Association. Ongoing multiorganizational meetings and structures devoted to addressing bias in general are needed. Racial and ethnic bias is one part of the picture, but other kinds of bias exist, such as bias toward LGBTQ patients, patients with obesity, patients with substance use disorders, and patients with psychiatric conditions in general. Broadening awareness of the different varieties of implicit bias can foster a culture of acceptance and inclusion.

If the expectation that modeling and mentoring by current minority faculty are the only ways to recruit Black women and men into psychiatry, the current situation will only get worse for the specialty over time. Psychiatry simply does not have the number of minority faculty to meet the existing demands, and asking those faculty to do more of the same leads to burnout, disillusionment, and eventually attrition from academic careers. It is the very definition of the "minority tax" [21]: asking underrepresented minority faculty to take on higher burdens and presence simply because they are minority. One strategy is to provide protected time and relief from clinical productivity expectations or other routine duties to perform these essential tasks in education, mentoring, recruitment, and advocacy.

All interested psychiatrists from a variety of practices, settings, and subspecialties can assist with mentoring. Academic departments will need to invest in programs that mentor underrepresented minority faculty [22], including Black men and women, and perhaps access outside funding sources to do so. It cannot be up to underrepresented medical faculty alone to shoulder this burden, given the barriers already in place. All in the academic department will need to emphasize and bring to life cultural humility.

We value the current academic diversity within our midst because we believe that interaction with individuals from varied backgrounds and life experiences provides opportunities to achieve unique and creative approaches to medical education, research, and clinical care [23]. Academic programs and universities should identify and address barriers to the recruitment and retention of Black men and women. True and unwavering commitment to inclusiveness in academic psychiatry and efforts to ensure diversity in the pipeline for the field will enrich and strengthen the profession of medicine in the generations to come. Such commitment and efforts will also demonstrate integrity and consistency: the values that govern clinical and advocacy work in psychiatry also are expressed in our dedication to welcoming all people to be our colleagues - our teachers, mentors, learners, and collaborators-working shoulder to shoulder and fulfilling our responsibilities to our patients, to society, and to one another in academic medicine.

\section{Compliance with Ethical Standards}

Conflict of Interest The authors declare that they have no conflict of interest.

\section{References}

1. Association of American Medical Colleges. U.S. Medical School Faculty, 2017. Available at https://www.aamc.org/data/ facultyroster/reports/486050/usmsf17.html. Accessed 12/31/18.

2. Liaison Committee on Medical Education. Functions and Structure of a Medical School. Available at http://lcme.org/publications/. Accessed 3/24/19.

3. Accreditation Council for Graduate Medical Education. Common Program Requirements (Residency). Available at https://www. acgme.org/Portals/0/PFAssets/ProgramRequirements/ CPRResidency2019.pdf. Accessed 12/31/18.

4. Association of American Medical Colleges. Underrepresented in Medicine. Available at https://www.aamc.org/initiatives/urm/. Accessed 3/24/19.

5. United States Census Bureau. Quick Facts: United States. Available at https://www.census.gov/quickfacts/fact/table/US/PST045218. Accessed 12/31/18.

6. Lett LA, Orji WU, Sebro R. Declining racial and ethnic representation in clinical academic medicine: a longitudinal study of 16 US medical specialties. PLoS One. 2018;13(11):e0207274.

7. Smedley BD, Stith Butler A, Bristow LR, Institute of Medicine. In the Nation's compelling interest: ensuring diversity in the health care workforce. Committee on institutional and policy-level strategies for increasing the diversity of the U.S. healthcare workforce. Washington DC: National Academies Press; 2004. Available at https://www.ncbi.nlm.nih.gov/books/NBK216009/pdf/Bookshelf NBK216009.pdf. Accessed 12/31/18.

8. IOM Report - Unequal Treatment: Confronting Racial and Ethnic Disparities in Health Care. 2002. Available at http://www. nationalacademies.org/hmd/Reports/2002/Unequal-TreatmentConfronting-Racial-and-Ethnic-Disparities-in-Health-Care.aspx. Accessed 1/1/19.

9. Griffith E, Jones B, Stewart A. Black mental health: patients, providers, and systems. Washington DC: American Psychiatric Association Publishing; 2019.

10. Crospey K, Masho SW, Shiang R, et al. Why do faculty leave? Reasons for attrition of women and minority faculty from a medical school: four-year results. J Women's Health (Larchmt). 2008;17(7): 1111-8.

11. Maina IW, Belton TD, Ginzberg S, Singh A, Johnson TJ. A decade of studying implicit racial/ethnic bias in healthcare providers using the implicit association test. Soc Sci Med. 2018;199:219-29.

12. UT Southwestern Medical Center Health Professions Recruitment and Exposure Program (HPREP). Available at https://www. utsouthwestern.edu/education/programs/nondegree-programs/ high-school-students/hprep/. Accessed 3/27/19.

13. Yale School of Medicine. Diversity, Inclusion, Community Engagement, and Equity (DICE). Health Professions Recruitment Exposure Program (High School). Available at https://medicine. yale.edu/dice/engagement/pipeline/hprep.aspx. Accessed 3/27/19.

14. Blueprint. Health Professions Recruitment and Exposure Program. Available at https://blueprint.uchicago.edu/organization/hprep. Accessed 4/1/19.

15. Smith R. Thoughts for new medical students at a new medical school. BMJ. 2003;327:1430-3.

16. Tsai J. Diversity and inclusion in medical school: the reality. Scientific American. July 12, 2018. Available at https://blogs. 
scientificamerican.com/voices/diversity-and-inclusion-in-medicalschools-the-reality/. Accessed 3/28/19.

17. Lu F, Primm A. Mental health disparities, diversity, and cultural competence in medical student education: how psychiatry can play a role. Acad Psychiatry. 2006;30:9-15.

18. Lokko H, Chen JA, Parekh RI, Stern TA. Racial and ethnic diversity in the US psychiatric workforce: a perspective and recommendations. Acad Psychiatry. 2016;40:898-904.

19. Taneja E, Sehgal P, Greenlee A, Frank AA. To dodge or disclose: a faculty development workshop to promote discussion of racial/ ethnic minority trainee cultural identity. Acad Psychiatry. 2019;43:239-43.

20. Harris TB, Mian A, Lomax JW, Scott-Gurnell K, Sargent JA, Phillips JL, et al. The Texas regional psychiatry minority Mentor network: a regional effort to increase psychiatry's workforce diversity. Acad Psychiatry. 2012;36:60-3.
21. Rodríguez JE, Campbell KM, Pololi LH. Addressing disparities in academic medicine: what of the minority tax? BMC Med Educ. 2015;15:6.

22. Beech B, Calles-Escandon J, Hairston KG, et al. Mentoring programs for underrepresented minority faculty in academic medical centers: a systematic review of the literature. Acad Med. 2013;88: 541-9.

23. Roberts LW, Maldonado Y, Coverdale JH, Balon R, Louie AK, Beresin EV. The critical need to diversify the clinical and academic workforce. Acad Psychiatry. 2014;38:394-7.

Publisher's Note Springer Nature remains neutral with regard to jurisdictional claims in published maps and institutional affiliations. 